\title{
Uso do Modelo TOPAZ para a Caracterização Fisiográfica da Bacia Hidrográfica do Açude Epitácio Pessoa
}

\author{
Josiclêda Domiciano Galvíncio \\ UFCG - josidg@dca.ufc.edu.br \\ Francisco de Assis Salviano de Sousa \\ DCA/UFPB -fassis@dca.ufcg.edu.br
}

Recebido: 11/08/03 revisado: 24/03/04 aceito: 20/12/04

\section{RESUMO}

Este trabalho é parte de um projeto de pesquisa que pretende examinar os impactos da variabilidade espacial e temporal da precipitação, do solo e da topografia na resposta do balanço bidrico de uma bacia bidrográfica. Atualmente, grande parte das técnicas usadas para análise de bacias hidrográficas deriva de parâmetros fisiográficos e utiliza como ferramenta os "Softwares" de Sistema de Informações Geográficas (SIG). Apesar de serem recentes, essas técnicas são enfadonhas, custam caro e consomem muito tempo. Em muitos casos são limitadas, por obterem parâmetros em apenas um nivel de detalhe ou escala. Como alternativa, aqui é utilizado o modelo de análise digital de terreno denominado TOP AZ (em inglês, TOpographic P ArameteriZation) para obter os parâmetros fisiográficos, necessários à maioria dos modelos hidrológicos.

Os resultados mostram que a bacia hidrográfica do acude Epitácio Pessoa possui doze sub-bacias com áreas maiores ou iguais a 4000 ha e comprimento minimo de rio de $50 \mathrm{~km}$. Este estudo revelou ainda que o terreno apresenta alta predominância na faixa de 0 a 2\% de declividade, abrangendo quase toda a bacia hidrográfica. Nas faixas superiores a 20\%, ocorrem áreas espacialmente dispersas em pequenas porcõos da bacia. A área da bacia hidrográfica em estudo é de aproximadamente 1089990 hectares. A direção do escoamento superficial se dá, em sua maioria entre 300 a $360^{\circ}$ e de 0 a $90^{\circ}$.

O programa TOP AZ mostrou-se uma ótima ferramenta alternativa para extração das características físicas da bacia hidrográfica em estudo.

\section{Palavras-chave: modelo TOPAZ; precipitação; balanço hídrico.}

\section{INTRODUÇÃO}

Segundo Villela \& Matos (1975) a declividade do solo de uma bacia hidrográfica controla, em boa parte, a velocidade com que se dá o escoamento superficial, afetando portanto o tempo que leva a água da chuva para concentrar-se nos leitos fluviais que constituem a rede de drenagem. A magnitude dos picos de enchente e a maior ou menor oportunidade de infiltração e susceptibilidade para erosão do solo dependem da rapidez com que ocorre o escoamento superficial na bacia.

Segundo Garbrecht \& Martz (1999) a parametrização topográfica pelo TOPAZ, envolve medidas de várias propriedades paramétricas do "raster" como derivações da rede de drenagem e translado em canais de sub-bacias. No "raster" são também armazenados os parâmetros espaciais, como elevações retificadas, inclinação de superfície do solo, aspectos do terreno, padrão e rede de drenagem e os limites de sub-bacias. Essas informações podem ser visualizadas por software externo apropriados. Em forma de matriz, por outro lado, são armazenados os parâmetros que descrevem características específicas da superfície do solo, como a geometria de canais ou de sub-bacias. Exemplos de parâmetros de rede de drenagem incluem: ordem e comprimento de canais, áreas de sub-bacias, densidade de drenagem, parâmetros para composição dessa rede e inclinação da área de drenagem, Garbrecht \& Martz (1997). Para cada uma das sub-bacias analisada apresenta-se aqui os seguintes parâmetros: área e rede de drenagem representativas, comprimento e inclinação do solo.

Exemplos de aplicações do TOPAZ podem ser vistos em Lacroix et al. (2002) que utiliza o modelo Semidistributed Land Use Runoff Process (SLURP) para discutir o uso da técnica de análise topográfica digital na parametrização desse modelo hidrológico. Em Bringne et al. (1997) com o modelo de poluição Agricultural Non Point Source (AGNPS), para desenvolver a interface entre o modelo AGNPS e o TOPAZ e em Seglenieks et al. (1998) que desenvolveram a interface entre o modelo hidrológico distribuído Watershed Flood (WATFLOOD) e o TOPAZ. De modo geral, usando-se o TOPAZ é possível derivar todas as características físicas de uma bacia hidrográfica, rapidamente, com ótima precisão e em dife- 
rentes níveis de detalhes; isto é, com número variável de sub-bacias.

\section{MATERIAL E MÉTODOS}

\section{Região de estudo}

A Figura 1 mostra a localização espacial, em relação à área geográfica do Estado da Paraíba, da bacia hidrográfica do açude Epitácio Pessoa.

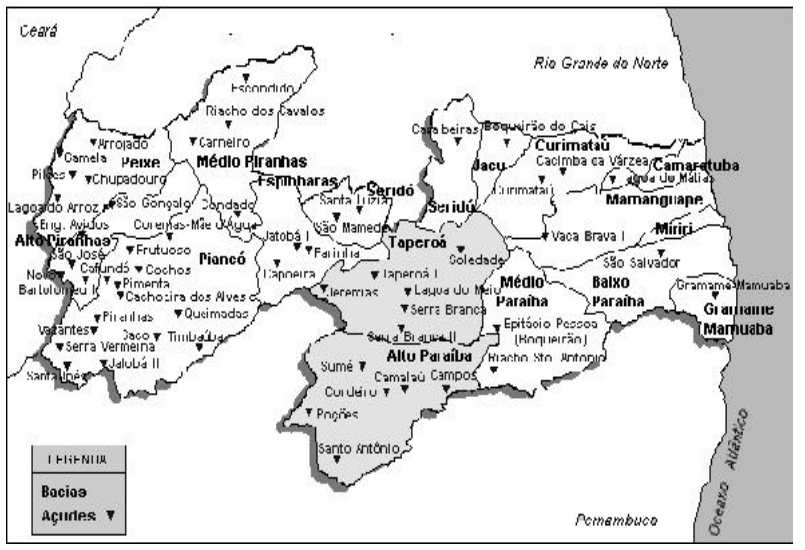

Figura 1 - Localização espacial (área em cinza) da bacia hidrográfica do açude Epitácio Pessoa

O clima característico da região em estudo é o semi-árido, quente e seco, segundo a classificação climática de Koëppen, com chuva de verão e com temperaturas máximas de $37^{\circ} \mathrm{C}$ e mínimas de $16^{\circ} \mathrm{C}$. A vegetação predominante da região é a caatinga. Os solos da região, segundo Andrade (1997), se caracterizam por serem rasos e, quase sempre, pedregosos. O tipo de solo mais comum encontrado na região é o Aluvial Estrófico.

A bacia de Boqueirão pertence à grande bacia do rio Paraíba, um dos mais importantes do Estado. Com relação aos recursos hídricos é pobre em seus amplos aspectos, de forma que sua produção de água é insuficiente para abastecer reservatórios em condições de longos períodos de estiagens.

\section{MATERIAL UTILIZADO}

O DTED (em inglês, Digital Terreno Elevation Data) é um arquivo que contém dados digitais de elevações do terreno, para vários pontos da superfície, com distâncias horizontais regularmente espaçadas. A National Imagery and Mapping Agency (NIMA) dos EUA, desenvolveu um padrão para um conjunto de dados digitais denominado de "DTED level 0" que pode ser utilizado por cientistas, técnicos e o público em geral. Esse DTED é uma matriz uniforme com valores de elevação do terreno. $\mathrm{O}$ "DTED level 0" disponibiliza informações de elevação, com espaçamento horizontal de 30 arc de segundo $(0,0083 \ldots$ graus $)$, que corresponde, aproximadamente, a um quilômetro. Esses dados de elevação podem ser adquiridos através de arquivos na forma binária, com valores mínimos, médios e máximos de elevações para cada uma das células, grátis e via internet na página da USGS (1998).

Neste trabalho, os DTED serão usados como a principal fonte de dados do programa TOPAZ, versão 3.1, do USDA (United States Department of Agriculture). Os DTED serão modelados e interpolados para gerar mapas digitais de elevações do terreno da bacia hidrográfica em estudo. Esse mapeamento digital de elevação do terreno geralmente toma como referência o nível médio do mar.

A parametrização automática da rede de drenagem da bacia hidrográfica em estudo foi baseada no conceito de simulação de fluxo sobre a superfície, O Callaghan \& Mark, (1984); Jenson \& Domingue (1988); Mark \& Dejong (1988) e Morris \& Heerdegen (1988). Esse conceito aplica o método D8, discutido em Fairchild \& Leymarie (1991), para determinar a direção do fluxo de cada célula. O método D8 compara a elevação local com as oito elevações de sua vizinhança. Dessa forma é possível determinar a direção do fluxo, das maiores para as menores elevações.

O comprimento de uma sub-bacia é definido como a distância entre a exutória e o ponto mais afastado. Em geral, as sub-bacias têm formatos irregulares, portanto pode haver vários possíveis valores de comprimentos, que por sua vez dependem da localização onde a estimativa é feita. Garbrecht \& Martz (1999) propõem dois métodos, denominado "Data Reduction" para calcular um comprimento de sub-bacia: 1) Distância média do deslocamento do curso de água e 2) Comprimento médio do percurso do rio. No primeiro método, o comprimento da sub-bacia é definido pela distância média do escoamento superficial, do seu ponto de origem, no interior da sub-bacia, até o primeiro canal adjacente de menor inclinação. Para obter essas estimativas, através dessas técnicas supracitadas, é necessário utilizar um Modelo de Elevação Digital (em inglês, DEM). Todas as informações necessárias para se calcular o comprimento do curso de água, tais como: contorno da sub-bacia, canais adjacentes e padrão de fluxo, podem ser obtidas pelo DEM. Aqui, o comprimento médio do curso de água é calculado pela média aritmética de todos os comprimentos dos cursos de água da sub-bacia. Cada uma das distâncias individual de curso d'água é calculada como a distâncias de curso de água é calculada, ao longo do rio, entre uma célula da sub-bacia e o canal adjacente. A distância média do deslocamento do curso de água é calculada pela expressão: 


$$
L_{t}=\frac{\sum_{i=1}^{n_{s}} D_{i} k_{i}}{\sum_{i=1}^{n_{s}} k_{i}}
$$

em que $L_{t}$ t é a distância média do curso d’água, $\mathrm{n}_{\mathrm{S}}$ é o número total de célula da sub-bacia, $\mathrm{D}_{\mathrm{i}}$ é a distância do curso d'água, da célula i para o canal adjacente e $\mathrm{k}_{\mathrm{i}}$ é um fator de ponderação, com valor igual um para curso d'água originado na célula da sub-bacia e igual a 0,5 para curso d'água originada na célula do canal.

O método 2 é diferente do método 1, visto que, por definição, o ponto inicial, pode ser considerado em qualquer célula da sub-bacia. É importante notar que esses divisores de drenagem não são apenas localizados no contorno da bacia. Os picos da topografia, no interior da subbacia, produzem, também, divisores de drenagem que representam pontos iniciais dos afluentes. Como resultado, esses divisores locais (os picos), geralmente, geram comprimento médio dos afluentes menor do que a distância média entre o contorno da sub-bacia e o canal adjacente. Comprimento médio do percurso do rio é estimado por:

$$
L_{f}=\frac{1}{n_{i}} \sum_{i=1}^{n_{i}} 1_{i}^{f}
$$

em que, $\mathrm{L}_{\mathrm{f}}$ é o comprimento médio do curso de água, $\mathrm{n}_{\mathrm{i}}$ é o número de afluentes na sub-bacia, i é um contador de afluentes e $\mathrm{l}^{\mathrm{f}}$ é o comprimento individual do afluente.

A declividade do terreno é definido por um plano tangente em um ponto da superfície. A declividade é composta por duas componentes: 1) o gradiente, as vezes também chamado de declividade, que é a máxima razão de variação de $\operatorname{cota} z$ e; 2) direção dessa máxima razão de variação de cota. A declividade pode ser expressa em graus $\left(0\right.$ a $\left.90^{\circ}\right)$ ou em porcentagem, enquanto que a direção é expressa em graus $\left(0\right.$ a $\left.360^{\circ}\right)$. As formulações gerais para o cálculo da declividade $\mathrm{D}$ e da direção E são dadas a seguir:

$$
\begin{aligned}
& \mathrm{D}=\operatorname{arctg}\left[\left(\frac{\partial \mathrm{Z}}{\partial \mathrm{X}}\right)^{2}+\left(\frac{\partial \mathrm{Z}}{\partial \mathrm{Y}}\right)^{2}\right]^{1 / 2} \\
& \mathrm{E}=\operatorname{arctg}\left[-\frac{\left(\frac{\partial \mathrm{Z}}{\partial \mathrm{Y}}\right)}{\left.\left(\frac{\partial \mathrm{Z}}{\partial \mathrm{X}}\right)\right] \quad(0<\mathrm{E}<2 \pi)}\right.
\end{aligned}
$$

em que $\frac{\partial z}{\partial x}$ e $\frac{\partial z}{\partial y}$, são derivadas parciais nas direções X e Y.
Segundo Burrough (1986), se a superfície for representada por um modelo de grade regular, a melhor estimativa das componentes do vetor gradiente, em uma posição i, j do modelo, é obtida por :

$\left[\frac{\partial Z}{\partial X}\right]_{i, j}=\left[\begin{array}{l}\left(Z_{i+1, j+1}+2 * Z_{i+1, j}+Z_{i+1, j-1}\right)- \\ \left(Z_{i-1, j+1}+2 * Z_{i-1, j}+Z_{i-1, j-1}\right)\end{array}\right] / 8 * \partial X$

$\left[\frac{\partial Z}{\partial Y}\right]_{i, j}=\left[\begin{array}{l}\left(Z_{i+1, j+1}+2 * Z_{i, j+1}+Z_{i-1, j+1}\right)- \\ \left(Z_{i+1, j+1}+2 * Z_{i, j-1}+Z_{i-1, j-1}\right)\end{array}\right] / 8 * \partial Y$

em que os elementos de $Z$ estão distribuídos segundo oito pontos vizinhos à derivada de $\mathrm{Z}$.

A inclinação da superfície do terreno é definida, geralmente, pelo decréscimo de elevação, do ponto mais alto para o mais baixo. No interior da sub-bacia, as irregularidades na topografia da superfície do solo produzem inclinações que variam com o local. O TOPAZ utiliza quatro métodos para estimar a inclinação da superfície do solo, são eles: 1) inclinação média do terreno, 2) inclinação média da distancia do curso do percurso do rio, 3) inclinação média do percurso e, 4) inclinação global. Esses quatro métodos possuem diferentes conceitos de inclinação.

No método 1, a inclinação do terreno é definida como a maior inclinação do ponto sobre a superfície. Seu valor é estimado tomando-se a derivada de uma superfície matemática que se aproxima da superfície topográfica local. A inclinação média do terreno é dada pelo valor médio de todos os valores de inclinação no interior da subbacia. Matematicamente é estimada por:

$$
S_{t}=\frac{1}{n_{S}} \underset{i=1}{n_{t}} s_{i}^{t}
$$

em que, $\mathrm{S}_{\mathrm{t}}$ é a inclinação média do terreno, $\mathrm{n}_{\mathrm{S}}$ é o número de células da sub-bacia e $S_{i}{ }_{i}$ é a inclinação do terreno na célula i. A inclinação da célula i é obtida pelo ajuste de uma superfície quadrática para produzir as elevações do modelo em cada célula de três por três. Como dito antes, a derivada da superfície matemática dá a inclinação do terreno e é tomada na célula central, segundo Martz \& Dejong (1987).

No método 2, a distância média de inclinação do curso de água principal é dada pela média das inclinações associadas às distâncias dos afluentes, discutido anteriormente. Assim: 


$$
\mathrm{S}_{\mathrm{c}}=\frac{1}{\mathrm{n}_{\mathrm{t}}} \sum_{\mathrm{i}=1}^{\mathrm{n}_{\mathrm{s}}} \mathrm{s}_{\mathrm{i}}^{\mathrm{c}}
$$

em que, $\mathrm{S}_{\mathrm{c}}$ é a inclinação média do curso de água principal, $\mathrm{n}_{\mathrm{S}}$ é o número de células da sub-bacia e $\mathrm{S}_{\mathrm{i}}^{\mathrm{c}}$ é a inclinação do afluente que inicia na célula i. A inclinação do curso de água na célula i é dada pela média de todas as inclinações ao longo do rio, entre a célula i na sub-bacia e o canal adjacente.

No método 3, a inclinação média do afluente é dada pela média das inclinações de todos os subafluentes da sub-bacia e é estimada por:

$$
\mathrm{S}_{\mathrm{f}}=\frac{1}{\mathrm{n}_{\mathrm{f}}} \sum_{\mathrm{i}=1}^{\mathrm{n}_{\mathrm{f}}} \mathrm{s}_{\mathrm{i}}^{\mathrm{f}}
$$

em que, $S_{f}$ é a inclinação média do afluente, $\mathrm{n}_{\mathrm{f}}$ é o número de subafluentes da sub-bacia e $\mathrm{S}_{\mathrm{i}}^{\mathrm{f}}$ é a inclinação do subafluente a partir do afluente na célula $\mathrm{i}$.

No método 4, a inclinação global é definida pela diferença entre a elevação média da sub-bacia e o canal adjacente, dividida pelo comprimento médio do curso de água.

$$
S_{g}=\frac{E_{s}-E_{c}}{L_{t}}
$$

em que $S_{g}$ é a inclinação global da sub-bacia, Es é a elevação média da sub-bacia, $\mathrm{E}_{\mathrm{C}}$ é a elevação média do canal adjacente e $\mathrm{L}_{\mathrm{t}}$ é o comprimento médio do curso de água.

Para realizar as tarefas descritas acima, que envolve grande quantidade de dados, é preciso que se use o compilador FORTRAN, para compilar e processar o TOPAZ e "softwares" como por exemplo, IDRISI, escolhido neste estudo para visualizar as saídas do TOPAZ.

\section{RESULTADOS E DISCUSSÃO}

Neste trabalho obteve-se, de forma automática, as seguintes características físicas da bacia hidrográfica em estudo: inclinação, direção do fluxo, comprimento dos cursos de água, áreas de drenagem da bacia e sub-bacias.

A Figura 2, mostra a delimitação automática com base no software TOPAZ. Aqui, a bacia hidrográfica em estudo foi dividida em doze sub-bacias. Essa divisão foi obtida fixando-se os parâmetros CSA (Critical Source Area) e MSCL (Minimum Source Channel Length). O critério para a escolha do CSA $=4000$ ha e do MSCL $=50$ $\mathrm{km}$ foi baseado na regionalização mensal da precipitação da bacia em estudo.

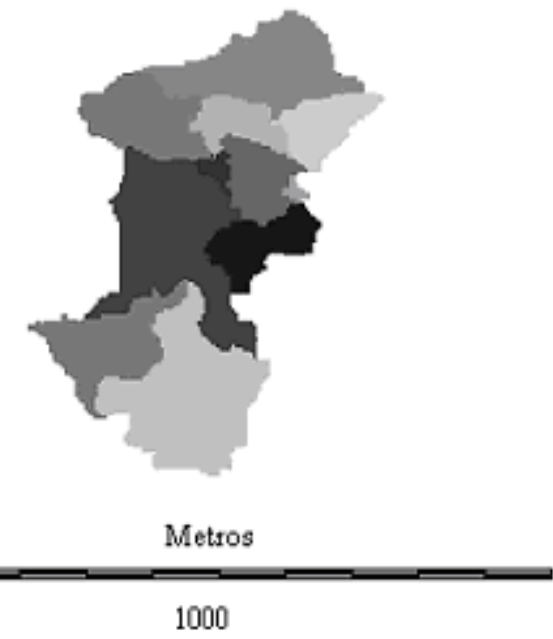

Figura 2 - Delimitações da bacia em estudo

A Figura 3 mostra a definição da rede de drenagem dentro do contorno da bacia hidrográfica, essa saída pode ser selecionada pelo o usuário durante a execução do programa. Quanto menor o valor do parâmetro CSA, tanto maior será a representação da rede de drenagem. Esse procedimento do programa serve para avaliar o nível de exigência da representação da rede de drenagem. Pode ser visto na Figura 3 que o valor do parâmetro CSA $=4000$ ha, representa bem a rede de drenagem da bacia em estudo. A rede de drenagem extraída pelo o TOPAZ permite o reconhecimento da disposição espacial dos elementos de drenagem, favorecendo a identificação das zonas homogêneas de drenagem e oferecendo subsídios para que se faça inferências a respeito de áreas de drenagem similares. Pode ser visto, ainda, na Figura 3 que a angularidade prevalecente é aguda, dando a essas drenagens padrões drendríticos. Isso sugere que a bacia em estudo apresenta solo raso, baixa capacidade de armazenamento de água no subsolo e baixas condições de fertilidade do solo.

A Figura 4 mostra o aspecto da inclinação da área que contém a bacia em estudo. Essa Figura resume, usando o método D8, a variação das elevações de cada uma das células. Assim, tem-se uma visão espacial das direções dos fluxos sobre o terreno. Essas direções são dadas em valores inteiros e positivos de 0 a 360 graus. $O$ aspecto do terreno é de grande importância para a identificação da direção do escoamento superficial e conseqüentemente na identificação das sub-bacias. Na Figura 4, nota-se que as direções do escoamento superficial se dar em sua maioria entre 300 a $360^{\circ}$ e 0 a $90^{\circ}$, ou seja, na direção sudoeste e nordeste da área do modelo digital de elevação, utilizado neste estudo. Justamente, na direção onde encontra-se situado o açude Epitácio Pessoa. 


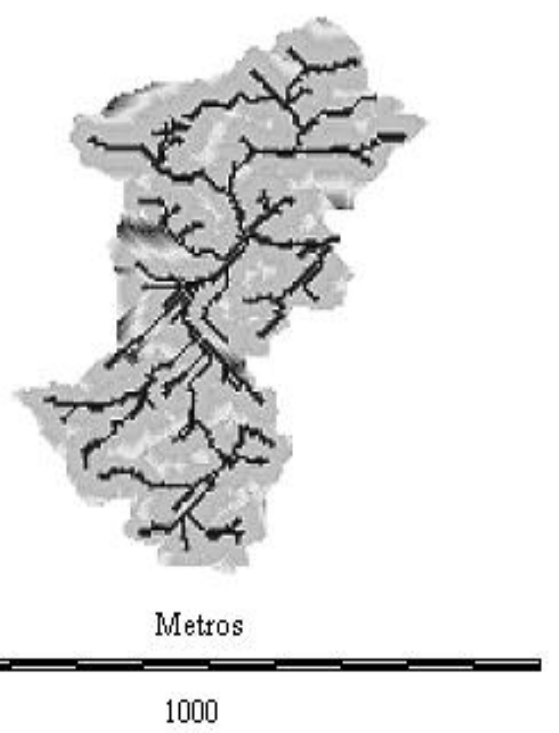

Figura 3 - Drenagem da bacia em estudo usando o TOPAZ

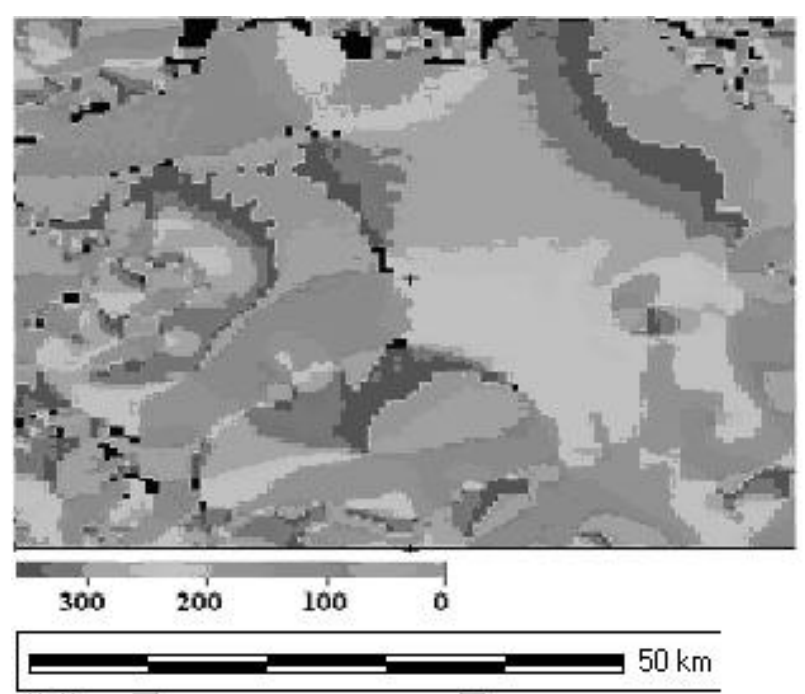

Figura 4- Aspectos da inclinação da área que contém a bacia em estudo

A Figura 5 apresenta a relação entre a inclinação média do terreno e a elevação em metros. Neste trabalho, a inclinação média do terreno, da área que contém a bacia em estudo, apresenta alta predominância da faixa de 0 a $2 \%$ de declividade, abrangendo quase toda a bacia hidrográfica do açude Epitácio Pessoa. Nas faixas superiores a $20 \%$, ocorrem áreas espacialmente dispersas, porém concentradas em pequenas porções da bacia. $O$ valor da inclinação média da bacia hidrográfica do açude Epitácio Pessoa é cerca de 0,47. Essa informação é de grande importância para a modelagem hidrológica, visto que esse parâ- metro influencia sobremaneira o processo de infiltração, a velocidade do escoamento superficial e a entrada e saída de água nas sub-bacias.

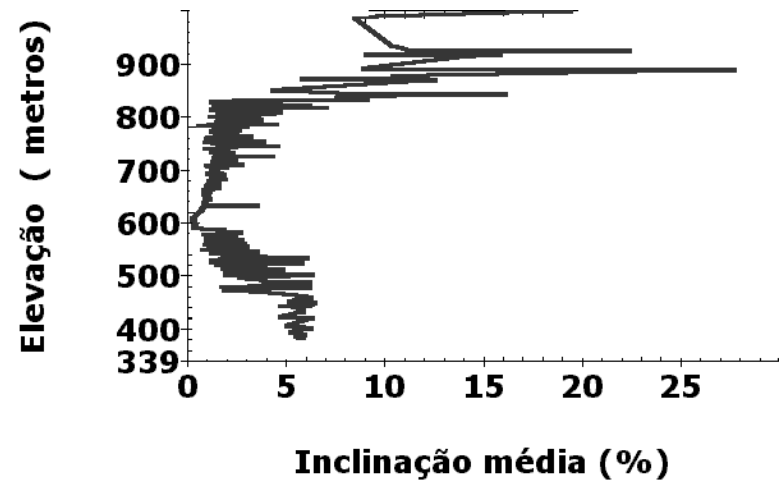

Figura 5- Relação inclinação versus elevação

Foram obtidas algumas estatísticas amostrais, dos valores de elevações do solo da área que contém a bacia, tais como: média, desvio-padrão, valor mínimo, valor máximo, e inclinação média. Esses valores podem ser vistos na Tabela 1.

Tabela 1 - Estatísticas descritivas dos dados

\begin{tabular}{lr}
\hline Média & 629,36 \\
\hline Desvio Padrão & 47,15 \\
Valor mínimo & 339,00 \\
Valor máximo & 1001,00 \\
Inclinação média do solo & 0,47 \\
\hline
\end{tabular}

A Tabela 2 apresenta os valores dos comprimentos dos canais principais e das áreas das sub-bacias. $\mathrm{Na}$ primeira linha da Tabela 2, o valor de 1089990 hectares, representa a área total da bacia hidrográfica do açude Epitácio Pessoa, obtida pelo programa TOPAZ. Na Tabela 2 a área de drenagem a montante é dada pela soma de todas as áreas de drenagem que estão acima de um ponto de controle. Já a área de drenagem direta é área que está ligada diretamente a saída da bacia.

A Tabela 3 apresenta os valores das inclinações das sub-bacias.

\section{CONCLUSÕES}

Com base nos resultados verifica-se que a bacia hidrográfica do açude Epitácio Pessoa apresenta doze subbacias com áreas maiores ou iguais a 4000 ha e comprimento mínimo de rio igual a $50 \mathrm{~km}$.

A bacia hidrográfica do açude Epitácio Pessoa possui uma área de aproximadamente 1089990 hectares.

A direção do escoamento superficial se dar em sua maioria entre 300 a $360^{\circ}$ e 0 a $90^{\circ}$. 
Tabela 2 - Informações de comprimentos e áreas das sub-bacias

\begin{tabular}{|c|c|c|c|c|c|}
\hline Sub-bacias & Ordem do canal & $\begin{array}{c}\text { Área total da sub- } \\
\text { bacia (hectares) }\end{array}$ & $\begin{array}{c}\text { Comprimento do } \\
\text { canal (metros) }\end{array}$ & $\begin{array}{l}\text { Área de drenagem a } \\
\text { montante (hectares) }\end{array}$ & $\begin{array}{c}\text { Área de drenagem } \\
\text { direta (hectares) }\end{array}$ \\
\hline 1 & 3 & - & - & 1089990 & - \\
\hline 2 & 2 & 195300 & 339500 & 894690 & 195300 \\
\hline 3 & 3 & 60570 & 182600 & 834120 & 60570 \\
\hline 4 & 1 & 53550 & 15700 & 50940 & 2610 \\
\hline 5 & 3 & 49140 & 68500 & 731430 & 49140 \\
\hline 6 & 2 & 174780 & 117900 & 264870 & 174780 \\
\hline 7 & 1 & 176310 & 186800 & 45540 & 130770 \\
\hline 8 & 1 & 88560 & 71400 & 66960 & 21600 \\
\hline 9 & 2 & 8100 & 45200 & 283680 & 8100 \\
\hline 10 & 1 & 80460 & 78400 & 62730 & 17730 \\
\hline 11 & 2 & 37170 & 77700 & 166050 & 37170 \\
\hline 12 & 1 & 117900 & 59900 & 44010 & 73890 \\
\hline 13 & 1 & 48150 & 21000 & 41040 & 7110 \\
\hline
\end{tabular}

Tabela 3 - Informação da inclinação das sub-bacias

\begin{tabular}{|c|c|c|c|c|c|c|c|c|}
\hline \multirow[t]{2}{*}{$\begin{array}{l}\text { Sub- } \\
\text { bacias }\end{array}$} & \multicolumn{2}{|c|}{$\begin{array}{l}\text { Inclinação média do terre- } \\
\text { no }\end{array}$} & \multicolumn{2}{|c|}{$\begin{array}{l}\text { Inclinação média do curso } \\
\text { d'água }\end{array}$} & \multicolumn{2}{|c|}{$\begin{array}{l}\text { Inclinação média do aflu- } \\
\text { ente }\end{array}$} & \multicolumn{2}{|c|}{ Inclinação Global } \\
\hline & Direito & Esquerdo & Direita & Esquerda & Direita & Esquerda & Direita & Esquerda \\
\hline 1 & 1,0170 & 0,2294 & 0,5094 & 0,1202 & 0,2736 & 0,1202 & 0,3920 & 0,0662 \\
\hline 2 & 2,3325 & 0,4697 & 2,0078 & 0,4163 & 0,5736 & 0,4163 & 0,7821 & 0,3270 \\
\hline 3 & 0,3781 & 2,1192 & 0,1226 & 1,3139 & 0,0776 & 1,3139 & 0,0942 & 0,6830 \\
\hline 4 & 0,2703 & 0,4221 & 0,0958 & 0,0167 & 0,1074 & 0,0167 & 0,1737 & 0,0109 \\
\hline 5 & 0,2344 & 0,0838 & 0,0528 & 0,0500 & 0,0399 & 0,0500 & 0,0757 & 0,0294 \\
\hline 6 & 1,0262 & 0,9195 & 0,4539 & 0,2486 & 0,3901 & 0,2486 & 0,5016 & 0,2756 \\
\hline 7 & 1,5474 & 0,1602 & 0,9070 & 0,0645 & 0,7241 & 0,0645 & 0,7711 & 0,0649 \\
\hline 8 & 0,3561 & 0,8884 & 0,2230 & 1,1111 & 0,1315 & 1,1111 & 0,1207 & 0,5514 \\
\hline 9 & 1,0198 & 0,1768 & 0,2055 & 0,0204 & 0,1373 & 0,0204 & 0,1639 & 0,0127 \\
\hline 10 & 0,2233 & 0,4041 & 0,0897 & 0,2226 & 0,0439 & 0,2226 & 0,0403 & 0,0738 \\
\hline 11 & 0,0079 & 1,1782 & 0,0333 & 1,1658 & 0,0333 & 1,1658 & 0,0441 & 0,4579 \\
\hline 12 & 0,4512 & 0,7040 & 0,3674 & 0,4258 & 0,3320 & 0,3005 & 0,3027 & 0,3281 \\
\hline 13 & 0,6340 & 0,0206 & 0,3478 & 0,0222 & 0,3559 & 0,0130 & 0,3740 & 0,0175 \\
\hline
\end{tabular}

Este estudo com o TOPAZ dará suporte a um modelo hidrológico para estimar o balanço hídrico sobre a bacia hidrográfica do açude Epitácio Pessoa.

\section{REFERÊNCIAS}

ANDRADE, A. R. S. de. Características Físico-Hidricas dos solos da Estação Experimental Algodão. Variabilidade Espacial. Dissertação de Mestrado apresentada ao curso de mestrado em Engenharia a Agrícola da Universidade Federal da Paraíba. Campina Grande, dezembro,1997.

BRINGNE, R. L.; Darden, R.W.; Theurer F. D.; Garbrecht, J. GIS-Based generation of AGNPS Water- shed Routing and Channel Parameters. ASAE Annual International Meeting, Mineapolis, Minnesota. pp. 29-32, 1997.

BurRough, P. A. Principles of Geographical Information Systems for Land Resources Assessment. Clarendon Press - Oxford- London. 1986.

FAIRCHILD, J.; LEYMARIE, P. Drainage Networks from Grid Digital Elevation Models. Water Resources Research. Vol. 27, $\mathrm{n}^{0}$ 04, pp. 29-32,1991.

GARBRECHT, J.; MARTZ, L.W. TOPAZ Version 1.20: An Automated Digital Landscape Analysis Tool for Topographic Evaluation, Identification, Watershed Segmentation and Subcatchment Parameterization Overview. Rep. \# 97-2, Grazinglands Research 
Laboratory, USDA, Agricultural Research Service, El Reno, Oklahoma, 21.p, 1997.

GARBRECHT, J., MARTZ, L.W. Digital Elevation Model Issues in Water Reasources Modeling. Presented at the $19 \mathrm{~h}$ ESRI International User Conference, Environmental Systems Research Institute. Published in the Proceedings of this Conference. San Diego, California, July, 26-30, 1999.

JENSON, S.K., DOMINGUE. J.O. Extracting Topographic Structure from Digital Elevation Data for Geographical Information System Analysis. Photogrammetric Engineering and Remote Sensing, v. 54 (11), p.p 1593-1600, 1988.

LACROIX, M. P.; MARTZ, L. W.; KITE, G. W.; GAR BRECHT, J. Using Digital Terrain Analysis Modeling Techniques for the Parameterization of a Hydrologic Model. Environmental Modelling \& Software. Vol.17, pp. 127-136, 2002.

MARK, L.W.; DEJONG, E. Using Cesium-137 to assess the Variability of Net Soil Erosion and its Association With Topography in a Canadian Prairie Landscape. Catena, vol.14(95): pp. 439-451, 1988.

MORRIS, D. G., HEERDEGEN, R. G. Automatically derived catchment boundary and channel networks and their hydrological applications. Geomorphology, 1 (2), 131- 141, 1988.

O'CALLAGHAM, J.F., MARTZ, D. M. The extraction of drainage networks from digital elevation data. Computer Vision, Graphic and Image Processing, 4 (3), p. 375-387, 1984.

SEGLENIEKES, F. SOULIS, E.D. KOUWEN, N. Developpment of automatic methods of obtaining the drainage and land cover databases for WATFLOOD. In: program and abstract, Scientific Meeting of the Canadian Geophysical Union, Quebec, Canada, p.10, 1998.

USGS d, Global 30 arc-second Elevation Data Set, http://edcwww.cr.usgs.gov/landdaac/gtopo30/ gtopo30.html as of March 1998.

VILLELA, S.M.; MATTOS, A. Hidrologia Aplicada. McGRAW-HILL, São Paulo,1975.
Use of the TOPAZ Model For the PhysiOgraphic Characterization of the Epitacio Pessoa Dam Watershed

\section{ABSTRACT}

This work is part of a research project to assess the impacts of the spatial-temporal variability of rainfall, soil and topography in a watershed water balance response. Nowadays, most of the techniques used for watershed analysis are derived from physiographic parameters and use Geographic Information Systems (GIS) softwares as a tool. Although they are recent, those techniques are boring, expensive and time-consuming. In many cases they are limited because they obtain parameters on a single level of detail or scale. As an alternative, the digital analysis terrain model (TOPAZ) is used here to obtain the physiographic parameters needed for most hydrologic models.

This study aims to present a TOPAZ model application in order to extract, automatically the physiographic parameters of the watershed of the Epitacio Pessoa dam.

The watershed was divided into twelve subwatersheds. The criteria for choice of critical source area, CSA $=4000$ ha and minimum source canal length, MSCL $=50 \mathrm{~km}$ were based on monthly rainfall regionalization of the watershed. This study also showed that the soil presents a high predominance at gradients ranging between 0 to $2 \%$, covering almost the whole watershed. Above $20 \%$, areas occur spatially dispersed in small portions of the watershed.

Key-words: TOPAZ Model, precipitation, water balance. 